\title{
Estimation of geometrical parameters for fan beam tomography
}

\author{
Grant T Gullberg $\dagger$, Benjamin M W Tsui $\ddagger$, Carl R Crawford $\S$ and \\ Eric R Edgerton $\ddagger$ \\ † Department of Radiology, Division of Medical Physics, University of Utah, Salt Lake \\ City, UT 84132, USA

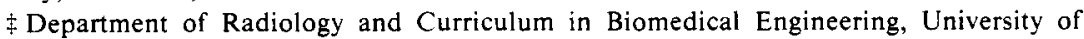 \\ North Carolina, Chapel Hill, NC 27514, USA \\ § Applied Science Laboratory, General Electric Medical Systems, Milwaukee, WI 53201, \\ USA
}

Received 5 January 1987, in final form 11 June 1987

\begin{abstract}
Tomographic image quality depends on precisely determining the geometric parameters that reference the detector system to the transaxial imaging coordinate system. For the parallel geometry, the projection of the centre of rotation onto the detector image plane completely defines the tomographic geometry. In addition to the projection of the centre of rotation onto the detector, fan beam geometry requires two other parameters that include the focal length and the projection of the focal point onto the detector. These parameters are related to the physical detector in a more complicated way than the projected centre of rotation required for parallel geometry. Heretofore no method has been developed for estimating the geometrical parameters of a fan beam detector system. A method is presented for estimating these parameters from centroids of the measured projections of a point source using the non-linear estimation algorithm due to Marquardt. The technique is applied to single photon emission computed tomography (SPECT) data from a rotating gamma camera using a fan beam collimator. The parameters can be determined very quickly in a clinical environment. The corresponding reconstructed images do not show image artefacts or loss of resolution characteristic of inaccurately determined geometric parameters.
\end{abstract}

\section{Introduction}

The fan beam geometry is used for most commercial X-ray CT scanners because of simplicity and speed of data acquisition. More recently, single photon emission computed tomography (SPECT) systems with rotating gamma cameras have been used which utilise fan beam collimators (Jaszczak et al 1979, Lim et al 1980, Tsui et al 1986) to improve sensitivity and resolution over those systems using parallel hole collimators. Reconstruction algorithms (Dreike and Boyd 1976, Herman and Naparstek 1977, Horn 1979, Weinstein 1980, Gullberg et al 1986) have been derived for fan beam detector systems. However, in order to obtain high-quality, artefact-free reconstructed images, it is necessary to precisely measure the geometrical parameters of the physically constructed detector system.

For parallel geometry, the projection of the centre of rotation onto the physical detector system is required. Analysis of SPECT systems with parallel geometry (Jaszczak et al 1981, Keyes et al 1982, Todd-Pokropek 1982) show that a misplaced centre of rotation will result in significant image artefacts. For SPECT systems the centre of rotation needs to be determined periodically in case there is any change in camera 
electronics. This is done by using either a point source or a line source and taking complementary views $180^{\circ}$ apart. Projections of the centre of rotation onto the image plane are determined for several complementary views by summing the centroids of the projected point source and dividing by two. The projection of the centre of rotation is then determined by averaging the calculated centroids from complementary views over several projection angles.

In this paper we will show that the geometrical parameters for a fan beam geometry can also be measured from projections of a point source. However, the measured projections are not a linear function of the geometrical parameters as in the parallel case. Therefore non-linear techniques are required to estimate the parameter values. For SPECT systems, the parameters need to be measured in the clinic immediately following a patient study and before the detector head has been moved for subsequent studies resulting in a new radius of rotation. For fan beam collimators it can happen that the centre of rotation does not align with the midline of the fan beam geometry. When data from a misaligned system are reconstructed with inaccurate measurements or completely ignoring the shift in the midline, there will be a loss of resolution for $360^{\circ}$ reconstruction (Kijewski and Judy 1983, Gullberg et al 1986). For halfscans, which are reconstructions from projections sampled over $180^{\circ}$ plus the fan angle, structured artefacts will result (Shepp et al 1979). Our approach is to include a shift parameter and to measure any misalignment in the centre of rotation. A reconstruction algorithm which includes this shift parameter is then used to reconstruct the fan beam projection data (Gullberg et al 1986).

A method for estimating the parameters of a fan beam detector system, including equations that relate the parameters to the centroid of the projections of a point source, is presented first, followed by a description of the Marquardt algorithm (Marquardt 1963) that is used to estimate the geometrical parameters. Results are presented for geometrical parameters estimated from data measured using a SPECT system with a fan beam collimator. These parameters are used to reconstruct slices for a temporomandibular joint (TMJ) study which is compared with results obtained using parameters that have been perturbed to show image artefacts.

\section{Formulation of the fan beam parameters}

The parameters of the fan beam geometry shown in figure 1 are the displaced centre of rotation $\tau$, focus-to-centre distance $D$, focus-to-detector distance $D^{\prime}$ and location, $c$, of the projection of the focus onto the detector. Mathematically, a point source

$$
f(x, y)=\delta\left(x-x_{0}\right) \delta\left(y-y_{0}\right)
$$

located at $\left(x_{0}, y_{0}\right)$ is used to develop a relationship between the parameters of the fan beam geometry shown in figure 1 and something that can be measured, namely the centroids of the projected point source. For multiple transaxial slices, the point source can actually be a line source perpendicular to the plane shown in figure 1 , so that all slices can be calibrated at the same time.

The projections of the point source are obtained by substituting equation (1) into the expression for the fan beam projection operator (Gullberg 1979):

$$
\begin{gathered}
R(\alpha, \xi)=\int_{-\infty}^{\infty} \int_{-\infty}^{\infty} f(x, y) \delta\left[(\xi-c)(x \sin \alpha-y \cos \alpha+D) / D^{\prime}\right. \\
-x \cos \alpha-y \sin \alpha+\tau] \mathrm{d} x \mathrm{~d} y
\end{gathered}
$$

where the projection coordinate $\xi$ denotes the distance from the edge of the measurable 


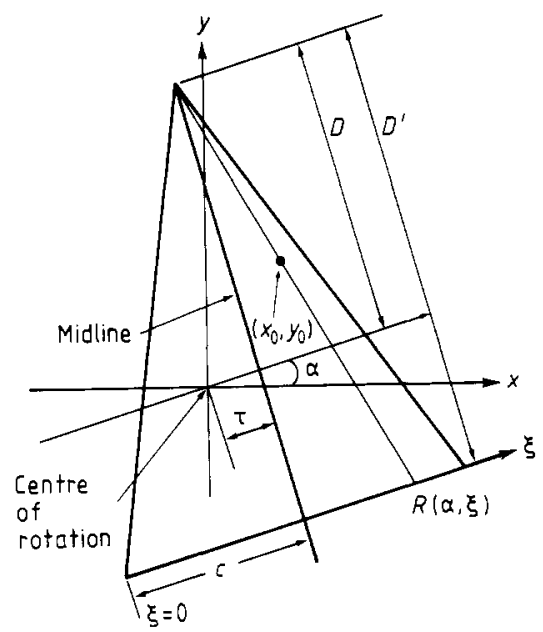

Figure 1. The parameters $x_{0}, y_{0}, c, \tau, D, D^{\prime}$ of the fan beam geometry are estimated by placing a point source at $\left(x_{0}, y_{0}\right)$ and obtaining data for various projection angles $\alpha$.

detector region and $\alpha$ is the projection angle for the geometry in figure 1. Performing this substitution gives the following expression for the projections of a point source:

$R(\alpha, \xi ; \beta)=\delta\left[(\xi-c)\left(x_{0} \sin \alpha-y_{0} \cos \alpha+D\right) / D^{\prime}-x_{0} \cos \alpha-y_{0} \sin \alpha+\tau\right]$

where $\beta=\left(\beta_{1}, \ldots, \beta_{6}\right)=\left(x_{0}, y_{0}, c, \tau, D, D^{\prime}\right)$ is a vector whose components are the parameters of the fan beam geometry plus the coordinates of the point source. For the angle $\alpha$, the centroid of a projection is $\rho(\beta, \alpha)$, defined by

$$
\rho(\beta, \alpha)=\left(\int_{-\infty}^{\infty} R(\alpha, \xi ; \beta) \xi \mathrm{d} \xi\right)\left(\int_{-\infty}^{\infty} R(\alpha, \xi ; \beta) \mathrm{d} \xi\right)^{-1} .
$$

Substituting equation (3) into equation (4) and integrating, we obtain

$$
\rho(\beta, \alpha)=D^{\prime}\left(x_{0} \cos \alpha+y_{0} \sin \alpha-\tau\right) /\left(x_{0} \sin \alpha-y_{0} \cos \alpha+D\right)+c .
$$

If the point source is located at the centre of rotation then the expression in equation (5) becomes independent of angle. In this case, it means that the centroid as a function of angle is independent of the parameters of the fan beam geometry.

The result in equation (5) gives an expression for the centroid of a projected point source in terms of the fan beam parameters. This suggests a method for estimating the geometrical parameters of a fan beam imaging system. In the experimental measurement, a point source is placed in the field of view of the scanner and away from the centre of rotation. Projections of the point source are collected and the centroid $\hat{\rho}_{i}$ is calculated for each angle $\alpha_{m}, m=1, \ldots, M$, using

$$
\hat{\rho}_{m}=\left(\sum_{n=1}^{N} \xi_{n} R\left(\alpha_{m}, \xi_{n}\right)\right)\left(\sum_{n=1}^{N} R\left(\alpha_{m}, \xi_{n}\right)\right)^{-1}
$$

where $R\left(\alpha_{m}, \xi_{n}\right), n=1, \ldots, N$, are the projection samples. For Poisson statistics, the variance $\operatorname{var}\left(\hat{\rho}_{m}\right)=\sigma_{m}^{2}$ for each centroid is determined from propagation of errors to give

$$
\begin{aligned}
\sigma_{m}^{2}=\left[\left(\sum_{n} \xi_{n}^{2} R\left(\alpha_{m}, \xi_{n}\right)\right)\left(\sum_{n} R\left(\alpha_{m}, \xi_{n}\right)\right)^{2}-\left(\sum_{n} \xi_{n} R\left(\alpha_{m}, \xi_{n}\right)\right)^{2}\left(\sum_{n} R\left(\alpha_{m}, \xi_{n}\right)\right)\right] \\
\times\left(\sum_{n} R\left(\alpha_{m}, \xi_{n}\right)\right)^{-4} .
\end{aligned}
$$


It is not hard to see that the expression in equation (7) is equal to the variance of the projected point source distribution divided by the number of samples taken.

The parameters of the fan beam geometry can be estimated by minimising the chi-squared function:

$$
\chi^{2}(\beta)=\sum_{m}\left[\hat{\rho}_{i}-\rho\left(\beta, \alpha_{i}\right)\right]^{2} \sigma_{m}^{-2}
$$

where $\rho\left(\beta, \alpha_{m}\right)$ is given in equation (5), $\hat{\rho}_{m}$ is the centroid calculated in equation (6) and $\sigma_{m}$ is the error in the calculation given in equation (7). The process of minimising equation (8) to determine estimates of the fan beam geometry is a non-linear estimation problem since the function $\rho(\beta, \alpha)$ in equation (5) is non-linear in the vector variable $\beta$.

The accuracy of the estimated fan beam parameters depends upon accurately measuring the location of the projected point source using the centroid calculation in equation (4) and, in particular, equation (6). The centroid calculation assumes that the detector system is uniform in sensitivity and resolution. We know by the very nature of the fan beam collimator with a flat detector that the sensitivity and resolution both vary as a function of depth and as a function of lateral sampling. To be more precise, the sensitivity variation should be compensated for and the resolution should be deconvolved before using the centroid calculation in equation (6). To prescribe the sensitivity variation for a converging collimator is not a trivial matter: the theory is complicated and, in addition, the theory usually does not correspond to the physical collimator. To make the sensitivity variation as small as possible, modifications to the collimator are made during the fabrication process by the manufacturer. This is desirable from the standpoint of having a uniform response across the field of view but makes it difficult to predict the actual sensitivity and resolution variations from the theory.

In practice, we have found that the centroid calculation in equation (6) works very well in calculating the parameters of the fan beam geometry even if corrections are not made for sensitivity or resolution variations. The reason for this is that the distribution of the projected point source is very localised. The resolution and sensitivity are nearly constant over the projected source distribution, therefore the centroid calculation in equation (6) gives a very accurate representation of the projection of the point source in the detector plane.

\section{The Marquardt algorithm}

Most methods that minimise equation (8) use successive approximations:

$$
\beta^{k+1}=\beta^{k}+\delta
$$

where either $\delta=\delta_{\mathrm{t}}$ is determined from a Taylor series expansion of $\rho$ or $\delta=\delta_{\mathrm{g}}$ is determined from a gradient $\chi^{2}(\beta)$ known as the steepest-descent method. The Taylor series correction is given by (Deutsch 1965)

$$
\delta_{\mathrm{t}}=B^{-1} E
$$

and the gradient correction is

$$
\delta_{\mathrm{g}}=2 E
$$


where the elements of the matrices $B$ and $E$ are defined by

$$
\begin{aligned}
& B=\left[b_{i j}\right]^{6 \times 6}=\left[\sum_{m=1}^{M} \frac{1}{\sigma_{m}^{2}} \frac{\partial \rho\left(\beta, \alpha_{m}\right)}{\partial \beta_{i}} \frac{\partial \rho\left(\beta, \alpha_{m}\right)}{\partial \beta_{j}}\right] \\
& E=\left[e_{i}\right]^{1 \times 6}=\left[\sum_{m=1}^{M} \frac{\partial \rho\left(\beta, \alpha_{m}\right)}{\partial \beta_{i}}\left(\hat{\rho}_{m}-\rho\left(\beta, \alpha_{m}\right)\right) / \sigma_{m}^{2}\right] .
\end{aligned}
$$

If we set

$$
\rho(\beta, \alpha)=\rho\left[\beta=\left(x_{0}, y_{0}, c, \tau, D, D^{\prime}\right), \alpha\right]=g / h+c
$$

where

$$
\begin{aligned}
& g=D^{\prime}\left(x_{0} \cos \alpha+y_{0} \sin \alpha-\tau\right) \\
& h=x_{0} \sin \alpha-y_{0} \cos \alpha+D
\end{aligned}
$$

the partial derivatives of $\rho(\beta, \alpha)$ in equations (12) and (13) are

$$
\begin{aligned}
& \partial \rho / \partial \beta_{1}=\partial \rho / \partial x_{0}=\left(D^{\prime} h \cos \alpha-g \sin \alpha\right) / h^{2} \\
& \partial \rho / \partial \beta_{2}=\partial \rho / \partial y_{0}=\left(D^{\prime} h \sin \alpha+g \cos \alpha\right) / h^{2} \\
& \partial \rho / \partial \beta_{3}=\partial \rho / \partial c=1 \\
& \partial \rho / \partial \beta_{4}=\partial \rho / \partial \tau=-D^{\prime} / h \\
& \partial \rho / \partial \beta_{5}=\partial \rho / \partial D=-g / h^{2} \\
& \partial \rho / \partial \beta_{6}=\partial \rho / \partial D^{\prime}=g /\left(h D^{\prime}\right) .
\end{aligned}
$$

The Taylor series method tends to diverge frequently and the steepest-descent methods are very slow to converge (Marquardt 1963). Marquardt found that the magnitude of the angle $\phi$ between the Taylor series correction vector $\delta_{\mathrm{t}}$ and the gradient vector $\delta_{\mathrm{g}}$ usually fell in the range $80^{\circ}<\phi<90^{\circ}$. It can be shown that a necessary requirement for any iterative method to converge is that the correction vector must be within $90^{\circ}$ of the gradient $\delta_{\mathrm{g}}$. To increase the speed of convergence of steepest descent methods, a more appropriate direction would be one which interpolates between the gradient $\delta_{\mathrm{g}}$ and $\delta_{\mathrm{t}}$. This is accomplished by solving for $\delta$ in the following equation:

$$
(B+\lambda I) \delta=E \text {. }
$$

Marquardt (1963) showed that as $\lambda \rightarrow \infty, \delta$ rotates toward $\delta_{\mathrm{g}}$. Therefore, in a region where the Taylor series method converges nicely, especially near the minimum, small values of $\lambda$ are chosen, whereas in regions where the Taylor series approximation is not adequate, $\lambda$ is increased so that $\delta$ approximates the gradient. The algorithm selects $\lambda$ to ensure that the optimum choice for $\delta$ is taken. The Marquardt algorithm has the especially useful feature that the direction and step size are determined simultaneously.

The Marquardt algorithm that was used by us is outlined in the appendix. Starting with an initial solution, the values of the geometric parameters are varied and the partial derivatives evaluated according to the algorithm to minimise the chi-squared function specified in equation (8). It is seen in equation (8) that the measured centroid of the projected point source is compared with the expression in equation (5). Thus one is effectively evaluating those parameters which make equation (5) come nearest to the measured centroid of the projected point source.

The estimated parameters have errors (Deutsch 1965) given by the covariance matrix

$$
\Phi=B^{-1}
$$


where the matrix $B$ in equation (12) is evaluated using the estimated parameters $\hat{\beta}$. The diagonal elements of the covariance matrix are the variances for the estimated parameters. The off-diagonal elements are the covariances which are a measure of the degree of correlation between the geometric parameters.

\section{Experimental results}

A typical study was performed to evaluate the technique for estimating the parameters of the fan beam collimator (Tsui et al 1986) shown in figure 2. The collimator was constructed with the dimensions shown in figure 3 . A line source of ${ }^{99} \mathrm{Tc}^{\mathrm{m}}$ was positioned parallel to the axis of rotation intersecting the transaxial plane at an unknown point, and the detector was rotated about its centre to take a set of 128 projections, similar to those illustrated in figure 4 . After the data were acquired, the centroid for each projection was determined using equation (6) and the measurement errors for the centroid calculations were determined using equation (7). The calculated centroids and errors are given in table 1 for every other angle that was sampled.

The data in table 1 have a discontinuity in the measured centroids between frame 123 and frame 1 . The reason for this discontinuity is probably due to variation in rotation angle between these frames. Variation in angular increments can occur on clinical SPEC $T$ systems. It is important that any errors in the angular increments are monitored and that they are within reasonable limits. The errors that occurred over a few angles in the data presented in table 1 did not seem to affect the estimation of the geometrical parameters.

Initial values in table 2 were assigned to each of the geometric parameters $x_{0}, y_{11}$, $c, \tau, D, D^{\prime}$. We have found good results if the coordinates $\left(x_{0}, y_{0}\right)$ of the point source are set initially to zero. The projection of the focal point onto the detector is usually

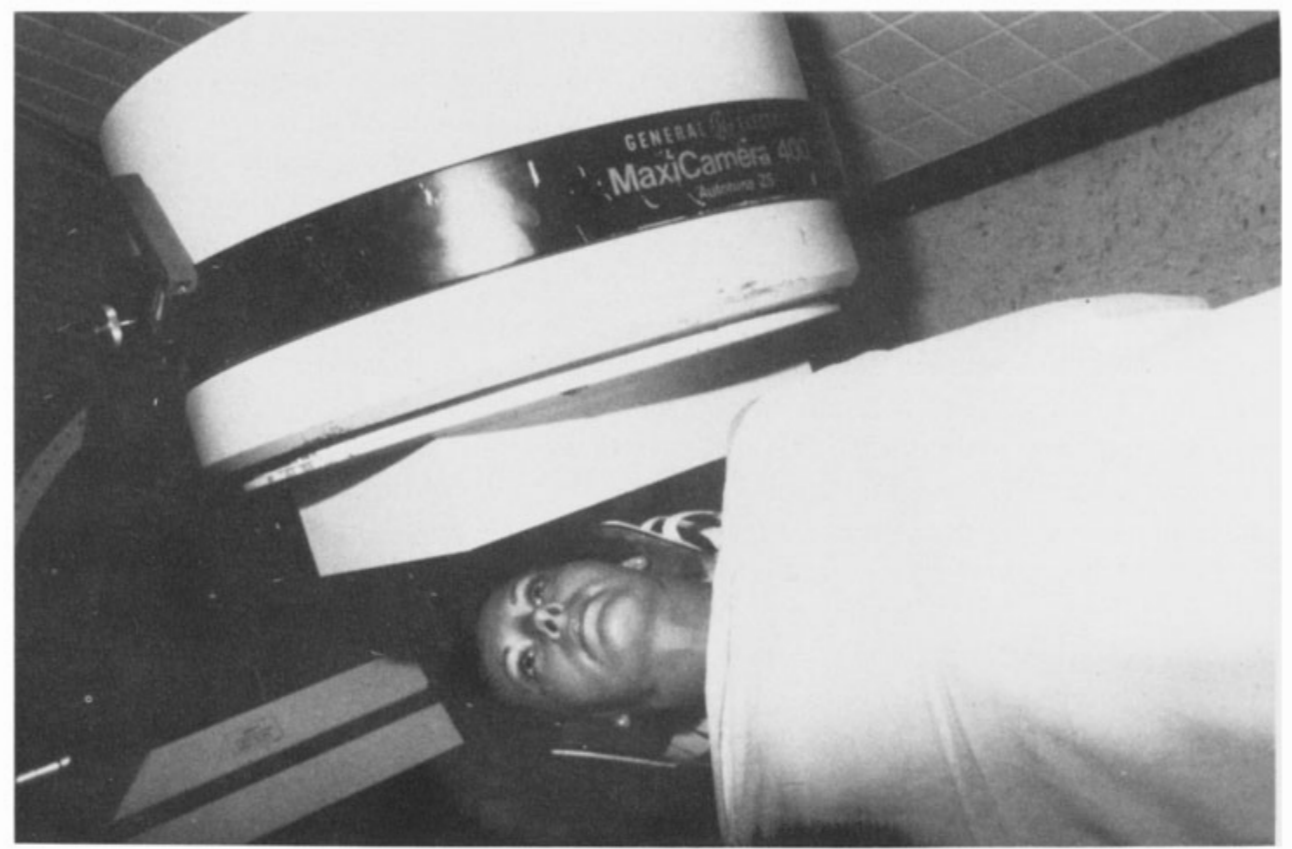

Figure 2. A spl: (I system with an extended fan beam collimator (Gencral Electric 400 ) $\mathrm{I}$, (jeneral H:Hectric Medical Systems, Milwaukee, WI). 


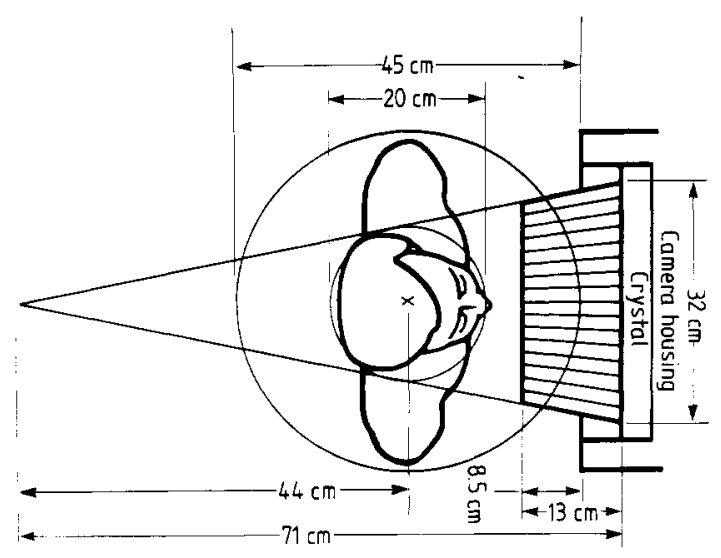

Figure 3. Approximate dimensions of the fan beam tomographic system shown in figure 2. The crystal is $40 \mathrm{~cm}$ in diameter and the projection of the focal point onto the crystal is approximately $20 \mathrm{~cm}$ from the edge of the crystal.

near the centre of the crystal at $c=20 \mathrm{~cm}$ and so this is a good initial guess. For the fan beam collimator that we used, the shift $\tau$ is very close to zero. The distance between the focal point and the centre of rotation $D$ will vary from study to study but usually will be approximately $44 \mathrm{~cm}$. The collimator was built to a designed specification for the focal length of $71 \mathrm{~cm}$ from the crystal face. Good results were obtained by initially choosing a focal length $D^{\prime}=60 \mathrm{~cm}$ and a distance between the focal point and the centre of rotation of $D=40 \mathrm{~cm}$. From simulations it has been found that the accuracy in the initial solution for $D^{\prime}$ is not as critical as it is for the other parameters. However, we have also noticed that an initial solution greater than $71 \mathrm{~cm}$ can give erroneous estimates of the focal length. Therefore a value of $D^{\prime}=60 \mathrm{~cm}$ was chosen as an initial guess for $D^{\prime}$. This is less than the design value but not so much that it would affect the convergence of the solution.

The geometric parameters were systematically and iteratively varied using the Marquardt algorithm to minimise the chi-squared function in equation (8). It can be found that many local minima are present which are not the true minimum being sought. To avoid settling on a local minimum, it is important to start with initial

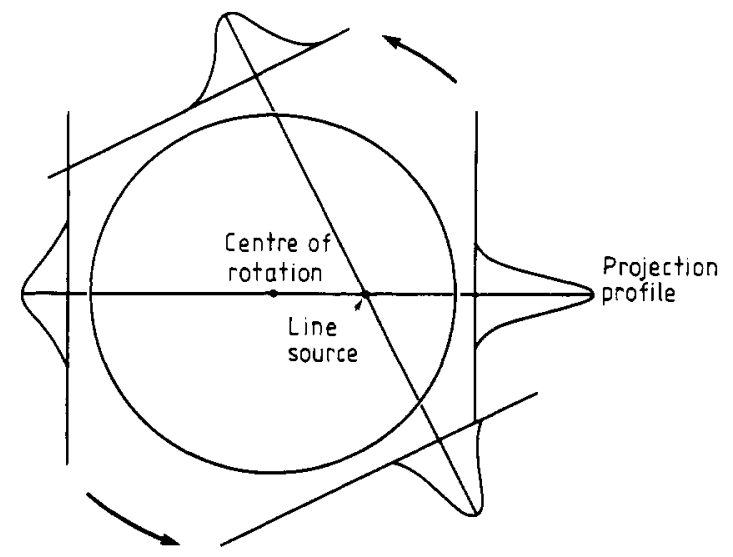

Figure 4. The projection of the line source will give profiles which will vary with the distance the line source is from the collimator. 
Table 1. Centroids (equation (6)) and errors (equation (7)) calculated from fan beam projection data of a line source of radioactivity using the SPECT system in figure 2 . The centroids are listed here for every other angle. The first angle is frame 1 and the last angle is frame 128.

\begin{tabular}{|c|c|c|c|c|c|c|c|}
\hline Frame & $\begin{array}{l}\text { Angle } \\
\text { (degrees) }\end{array}$ & $\begin{array}{l}\text { Centroid } \\
\text { point } \\
(\mathrm{cm})\end{array}$ & $\begin{array}{l}\text { Errors } \\
(\mathrm{cm})\end{array}$ & Frame & $\begin{array}{l}\text { Angle } \\
\text { (degrees) }\end{array}$ & $\begin{array}{l}\text { Centroid } \\
\text { point } \\
(\mathrm{cm})\end{array}$ & $\begin{array}{l}\text { Errors } \\
(\mathrm{cm})\end{array}$ \\
\hline 1 & 0 & 27.0867 & 0.0265 & 65 & 180.0 & 14.8689 & 0.0334 \\
\hline 3 & 5.6 & 26.9421 & 0.0310 & 67 & 185.6 & 14.8968 & 0.0327 \\
\hline 5 & 11.2 & 26.7923 & 0.0272 & 69 & 191.2 & 14.9787 & 0.0266 \\
\hline 7 & 16.9 & 26.5441 & 0.0341 & 71 & 196.9 & 15.1766 & 0.0357 \\
\hline 9 & 22.5 & 26.3174 & 0.0304 & 73 & 202.5 & 15.3747 & 0.0328 \\
\hline 11 & 28.1 & 25.9459 & 0.0316 & 75 & 208.1 & 15.6200 & 0.0350 \\
\hline 13 & 33.8 & 25.5971 & 0.0299 & 77 & 213.8 & 15.8961 & 0.0278 \\
\hline 15 & 39.4 & 25.2462 & 0.0266 & 79 & 219.4 & 16.2648 & 0.0297 \\
\hline 17 & 45.0 & 24.8148 & 0.0289 & 81 & 225.0 & 16.6640 & 0.0303 \\
\hline 19 & 50.6 & 24.3503 & 0.0303 & 83 & 230.6 & 17.1514 & 0.0303 \\
\hline 21 & 56.2 & 23.8860 & 0.0330 & 85 & 236.2 & 17.6163 & 0.0300 \\
\hline 23 & 61.9 & 23.3891 & 0.0281 & 87 & 241.9 & 18.1360 & 0.0278 \\
\hline 25 & 67.5 & 22.8630 & 0.0290 & 89 & 247.5 & 18.7094 & 0.0295 \\
\hline 27 & 73.1 & 22.3850 & 0.0288 & 91 & 253.1 & 19.3683 & 0.0299 \\
\hline 29 & 78.8 & 21.8426 & 0.0245 & 93 & 258.8 & 19.9768 & 0.0316 \\
\hline 31 & 84.4 & 21.2664 & 0.0296 & 95 & 264.4 & 20.6482 & 0.0325 \\
\hline 33 & 90.0 & 20.7201 & 0.0261 & 97 & 270.0 & 21.3146 & 0.0295 \\
\hline 35 & 95.6 & 20.1575 & 0.0323 & 99 & 275.6 & 22.0239 & 0.0301 \\
\hline 37 & 101.3 & 19.6040 & 0.0244 & 101 & 281.2 & 22.6515 & 0.0308 \\
\hline 39 & 106.9 & 19.0749 & 0.0274 & 103 & 286.9 & 23.1821 & 0.0309 \\
\hline 41 & 112.5 & 18.5642 & 0.0251 & 105 & 292.5 & 23.8897 & 0.0292 \\
\hline 43 & 118.1 & 18.0395 & 0.0321 & 107 & 298.1 & 24.4304 & 0.0334 \\
\hline 45 & 123.8 & 17.5634 & 0.0321 & 109 & 303.8 & 24.9231 & 0.0332 \\
\hline 47 & 129.4 & 17.1450 & 0.0314 & 111 & 309.4 & 25.4611 & 0.0310 \\
\hline 49 & 135.0 & 16.6912 & 0.0306 & 113 & 315.0 & 25.9243 & 0.0302 \\
\hline 51 & 140.6 & 16.2899 & 0.0283 & 115 & 320.6 & 26.3162 & 0.0314 \\
\hline 53 & 146.3 & 15.8752 & 0.0272 & 117 & 326.2 & 26.6289 & 0.0319 \\
\hline 55 & 151.9 & 15.6452 & 0.0318 & 119 & 331.9 & 26.9244 & 0.0328 \\
\hline 57 & 157.5 & 15.3593 & 0.0338 & 121 & 337.5 & 27.1533 & 0.0315 \\
\hline 59 & 163.1 & 15.1496 & 0.0323 & 123 & 343.1 & 27.2806 & 0.0293 \\
\hline 61 & 168.8 & 14.9603 & 0.0289 & 125 & 348.8 & 27.3272 & 0.0284 \\
\hline 63 & 174.4 & 14.8871 & 0.0326 & 127 & 354.4 & 27.3432 & 0.0279 \\
\hline
\end{tabular}

estimates which are as close to the actual value as possible, either obtained from measurements or from previous experience, as described above. It has also been found useful to vary only certain of the parameters at first to increase their precision before varying others. The results in table 2 first varied $x_{0}, y_{0}$ only, holding the other geometric parameters constant. The next pass varied $c$ only and then progressively varied different combinations of parameters.

Simultaneously varying the shift $\tau$, the focus-to-centre distance $D$ and the focus-todetector distance $D^{\prime}$ resulted in unrealistic values for the geometric parameters. In our case, the focal length of the collimator is long and, as such, the geometry is approaching that of a parallel beam causing the shift parameter $\tau$ and the focal length to become (i) more uncoupled from the problem and (ii) more different in absolute numerical value, and thus difficult for the algorithm to accurately determine their values. For shorter focal length collimators, the fitting technique becomes more sensitive to variations in these parameters. In general, the fitting technique most 
Table 2. The data in table 1 gave the following estimates for the geometrical parameters of the SPECT system with the fan beam collimator in figure 2 which was built to the design specifications given in figure 3 . In each pass, those variables indicated with an asterisk are held constant and the other variables are allowed to vary using the Marquardt algorithm to give a best estimate of the parameter. The errors for each estimate are given in parentheses and all values are in units of $\mathrm{cm}$.

\begin{tabular}{|c|c|c|c|c|c|c|c|}
\hline Pass & $x_{0}$ & $y_{0}$ & $c$ & $\tau$ & $D$ & $D^{\prime}$ & $x^{2}$ \\
\hline Initial value & 0 & 0 & 20 & 0 & 40 & 60 & \\
\hline 1 & $\begin{array}{c}4.0752 \\
( \pm 0.0016)\end{array}$ & $\begin{array}{c}-0.1844 \\
( \pm 0.0016)\end{array}$ & $20^{*}$ & $0^{*}$ & $40^{*}$ & $60^{*}$ & 362093 \\
\hline 2 & $4.0752^{*}$ & $-0.1844^{*}$ & $\begin{array}{c}21.0153 \\
( \pm 0.0017)\end{array}$ & $0^{*}$ & $40^{*}$ & $60^{*}$ & 2538 \\
\hline 3 & $4.0752^{*}$ & $-0.1844^{*}$ & $21.0153^{*}$ & $\begin{array}{c}-0.0024 \\
( \pm 0.0037)\end{array}$ & $40^{*}$ & $60^{*}$ & 2533 \\
\hline 4 & $4.0752^{*}$ & $-0.1844^{*}$ & $21.0153^{*}$ & $-0.0024^{*}$ & $\begin{array}{c}39.8882 \\
( \pm 0.0157)\end{array}$ & $60^{*}$ & 2483 \\
\hline 5 & $\begin{array}{c}4.0729 \\
( \pm 0.0006)\end{array}$ & $\begin{array}{c}-0.2280 \\
( \pm 0.0015)\end{array}$ & $\begin{array}{c}21.0140 \\
( \pm 0.0017)\end{array}$ & $-0.0024 *$ & $\begin{array}{c}39.8821 \\
( \pm 0.0157)\end{array}$ & $60^{*}$ & 1700 \\
\hline 6 & $\begin{array}{c}3.8257 \\
( \pm 0.0016)\end{array}$ & $\begin{array}{c}-0.2140 \\
( \pm 0.0017)\end{array}$ & $\begin{array}{c}21.0137 \\
( \pm 0.0020)\end{array}$ & $-0.0024^{*}$ & $\begin{array}{c}42.3748 \\
( \pm 0.0184)\end{array}$ & $\begin{array}{c}67.9340 \\
( \pm 0.0320)\end{array}$ & 1463 \\
\hline
\end{tabular}

accurately determines the centre of rotation c. Various initial solutions were tested and, in all cases, $c$ converged to a value very close to $21.0 \mathrm{~cm}$. Of the parameters, the focal length $D^{\prime}$ is probably the one least accurately determined by the estimation technique. We also found that fixing the focal length to the design value of $71 \mathrm{~cm}$ and estimating the other parameters gave reasonable results for the estimated parameters. These parameters also gave reconstructions with good image quality. In some cases, for initial solutions far from the solution, the estimated parameters converged to values that do not correspond in any way to the specific design of the collimator. However, these parameters can give reasonable reconstructed image quality. This happens with parameters that are highly correlated, such as $\tau$ and $c$. This is not very appealing from the standpoint of being able to determine experimentally the design parameters of the collimator. Therefore we have found that, using the initial solutions suggested above, reasonable estimates for the geometrical parameters and good reconstructed image quality are obtained.

The estimated parameters in table 2 after pass 6 were used to reconstruct data from a TMJ patient study. The patient was given $555 \mathrm{MBq}(15 \mathrm{mCi})$ of ${ }^{99} \mathrm{Tc}^{\mathrm{m}}$ labelled HDP intravenously and, $3 \mathrm{~h}$ later, projection data were obtained. The projection data were digitised in $64 \times 64$ matrices giving a pixel size of $3.8 \mathrm{~mm}$ at the axis of rotation. The slice thickness was $6.4 \mathrm{~mm}$. A total of 128 views over $360^{\circ}$ around the patient's head were collected. Figure 5 shows the reconstructed transaxial images of the TMJ. Increased uptake is seen in the right TMJ showing that the lateral aspect of the TMJ is involved.

Reconstructions with incorrect geometric parameters are given in figure 6 for the same transaxial slice as in figure $5(d)$. In figure $6(a)$, the projection of the focal length onto the detector, $c$, was set to $21.33 \mathrm{~cm}$ with the other parameters set to their corresponding values given in table 2 after pass 6 . In the same manner, in figure $6(b)$ the shift $\tau$ was set to $0.32 \mathrm{~cm}$, and in figures $6(c)$ and $6(d)$, the focal length $D^{\prime}$ was set to 54.36 and $78.13 \mathrm{~cm}$, respectively. These values for the geometric parameters were determined by performing a series of reconstructions that varied the parameter 

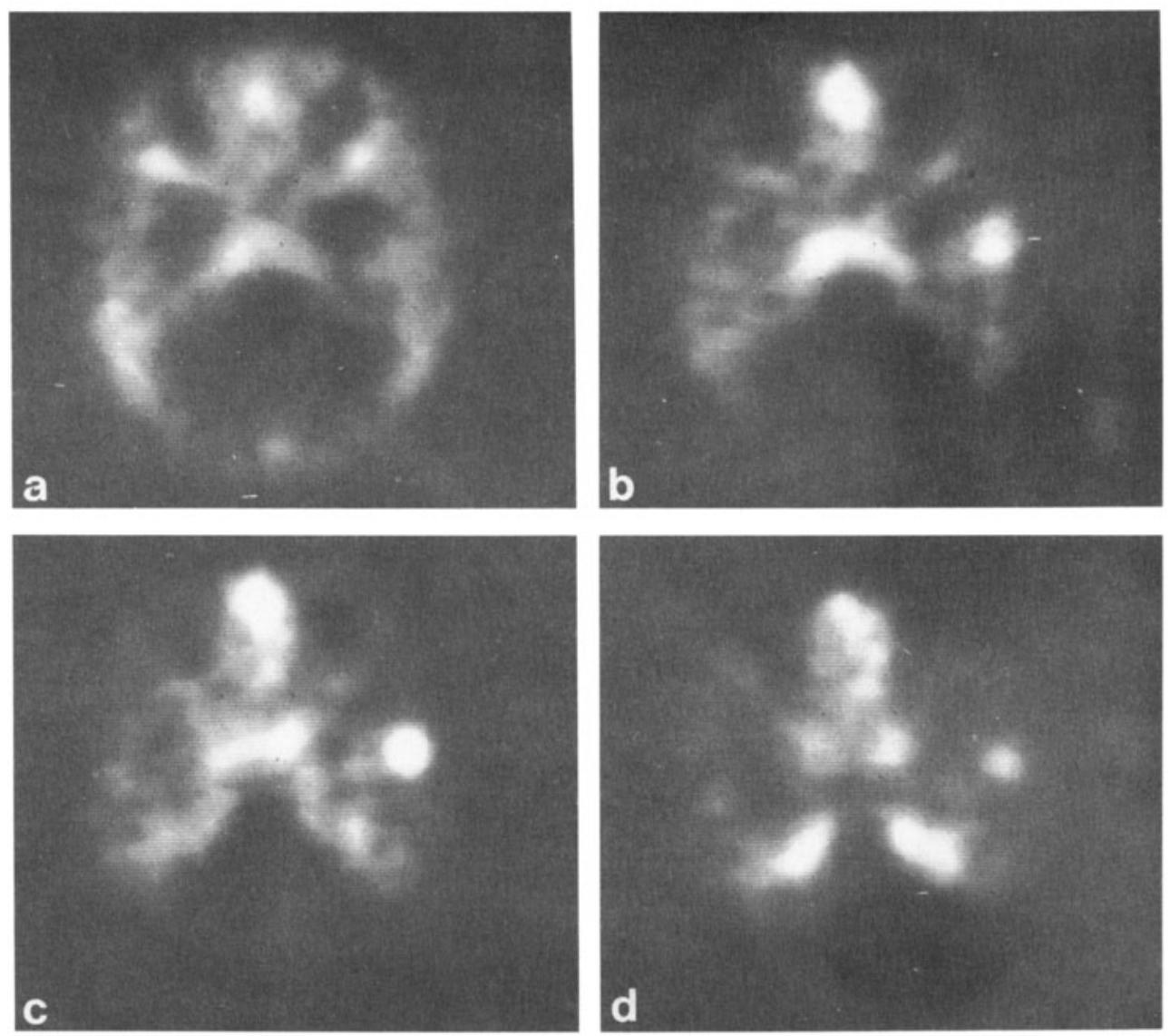

Figure 5. Reconstructed images from a patient with right ral disease, using the spl:c system with the extended fan beam collimator shown in figure 2 and the estimated parameters in table 2 .

of interest while keeping the other parameters set to the estimated values given in table 2. The examples in figure 6 are for those values for which the reconstruction began to show noticeable image artefacts. It can be seen that the image quality is very sensitive to the projection of the focal length onto the detector and the shift. The image quality is less sensitive to variations in the focal length for the particular collimator that was studied. It is interesting to note that image quality is more sensitive to errors in $D^{\prime}$ that are greater than the designed focal length than errors that are less. Reconstructions were not performed for different values of $D$, the distance between the focal point and the centre of rotation. The selection of $D$ only scales the reconstructed image and does not in any way distort the reconstruction.

\section{Discussion and conclusion}

We have presented a method where the parameters of a fan beam detector system can be determined using a non-linear estimation technique that estimates the geometrical parameters from projection measurements of a point source. The method has been proven to work efficiently in a clinical environment for SPI. C $T$ imaging where immediately following each patient study the fan beam parameters are calculated using a 

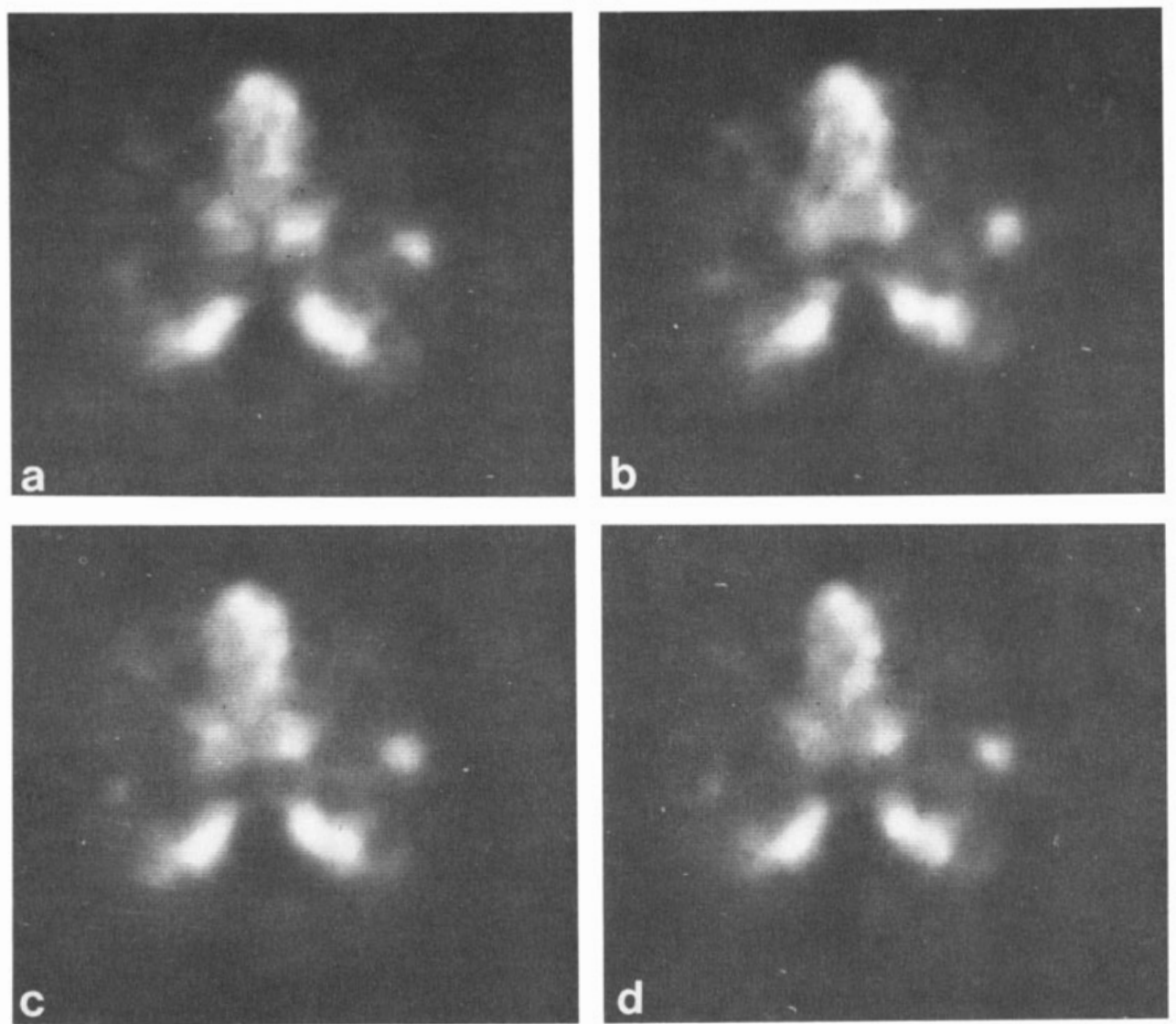

Figure 6. Reconstructed images of the same transaxial slice shown in figure $5(d)$ with geometrical parameters set to incorrect alues. $(a) c=21.33 \mathrm{~cm},(b) \tau=0.32 \mathrm{~cm},(c) D^{\prime}=54.36 \mathrm{~cm}$ and $(d) D^{\prime}=78.1 .3 \mathrm{~cm}$.

point source or a line source of radioactivity and collection of $360^{\circ}$ of projection data. Careful attention has to be paid to the initial estimates used for the Marquardt algorithm and in routine clinical use can be determined from simple measurements and past experience of best estimates. The approach we have presented is also appropriate for calibrating $\mathrm{X}$-ray (T scanners. The estimation technique.can also be used for other types of geometries, such as cone beam (Jaszczak et al 1986), by properly defining the projection equations for a point source placed in the field of view.

\section{Acknowledgments}

We would like to thank Dr Ronald Huesman for the many discussions concerning the implementation of the Marquardt algorithm. The work presented in this manuscript was partially supported by General Electric Medical Systems.

\section{Appendix}

The Marquardt algorithm used to estimate the fan beam parameters is outlined as follows. 
(1) Select an initial solution $\beta_{0}$ and an initial value for $\lambda$ (say $\lambda=0.01$ ) and let $v=10$ and set $\beta=\beta_{0}$.

(2) Evaluate the least-squares function $\chi^{2}(\beta)$, given in equation (8).

(3) Evaluate the matrices $B$ and $E$ from equations (12) and (13).

(4) Evaluate the matrices $B^{*}$ and $E^{*}$ where

$$
\begin{aligned}
& b_{i j}^{*}=b_{i j} /\left(b_{i i} b_{j j}\right)^{1 / 2} \\
& e_{i}^{*}=e_{i} /\left(b_{i i}\right)^{1 / 2} .
\end{aligned}
$$

(5) Solve for $\delta_{1}^{*}$ and $\delta_{2}^{*}$ where

$$
\begin{aligned}
& \delta_{1}^{*}=\left(B^{*}+\lambda_{1} I\right)^{-1} E^{*} \\
& \delta_{2}^{*}=\left(B^{*}+\lambda_{2} I\right)^{-1} E^{*}
\end{aligned}
$$

and $\lambda_{1}=\lambda$ and $\lambda_{2}=\lambda / v$.

(6) Evaluate $\delta_{1}$ and $\delta_{2}$ with components given by

$$
\begin{aligned}
& \delta_{1 i}=\delta_{1 i}^{*} /\left(b_{i i}\right)^{1 / 2} \\
& \delta_{2 i}=\delta_{2 i}^{*} /\left(b_{i i}\right)^{1 / 2} .
\end{aligned}
$$

(7) Evaluate $\beta_{1}$ and $\beta_{2}$ where

$$
\begin{aligned}
& \beta_{1}=\beta+\delta_{1} \\
& \beta_{2}=\beta+\delta_{2} .
\end{aligned}
$$

(8) Evaluate $\chi_{1}^{2}=\chi^{2}\left[\beta_{1}\left(\lambda_{1}\right)\right]$ and $\chi_{2}^{2}=\chi^{2}\left[\beta_{2}\left(\lambda_{2}\right)\right]$ as in step 2 .

(9) If $\chi_{1}^{2} \leqslant \chi^{2}$ then let

$$
\lambda=\lambda_{1} \quad \beta=\beta_{1} \quad \delta=\delta_{1}
$$

and go to step 10 , else if $\chi_{1}^{2}>\chi^{2}$ and $\chi_{2}^{2}<\chi^{2}$ then let

$$
\lambda=\lambda_{2} \quad \beta=\beta_{2} \quad \delta=\delta_{2}
$$

and go to step 10, else go to step 11 .

(10) Test for convergence: if

$$
\left|\delta_{i}\right| /\left(\gamma+\left|\beta_{i}\right|\right)<\varepsilon \quad \text { for all } i
$$

then stop; else return to step 2. Marquardt suggested that possible values for $\gamma$ and $\varepsilon$ might be $\gamma=10^{-3}$ and $\varepsilon=10^{-5}$.

(11) Let $\beta_{3}=\beta$.

(12) Let $\lambda_{3}=v \lambda$.

(13) Evaluate $\delta_{3}^{*}$ where

$$
\delta_{3}^{*}=\left(B^{*}+\lambda I\right)^{-1} E \text {. }
$$

(14) Evaluate $\delta$ where

$$
\delta_{3 i}=\delta_{3 i}^{*} /\left(b_{i i}\right)^{1 / 2} .
$$

(15) Evaluate $\beta_{3}$ where $\beta_{3}=\beta_{3}+\delta$.

(16) Evaluate $\chi_{3}^{2}=\chi^{2}\left[\beta_{3}(\lambda)\right]$ as in step 2 .

(17) If $\chi_{3}^{2}<\chi^{2}$ then let

$$
\lambda=\lambda_{3} \quad \beta=\beta_{3} \quad \delta=\delta_{3}
$$


and go to step 10 , else if

$$
\left|\delta_{3 i}\right| /\left(\gamma+\left|\beta_{3 i}\right|\right)<\varepsilon \quad \text { for all } i
$$

then stop; else go to step 12 .

For an initial solution $\beta_{0}$, the Marquardt algorithm converges to a local minimum. The initial choice of $\lambda=0.01$ and $v=10$ was suggested by Marquardt (1963). If necessary, $\lambda$ is decreased by a factor $1 / v$ in step 5 or increased by a factor $v$ in step 12 in order that an optimum choice of $\lambda$ is made which satisfies $\chi_{n+1}^{2}<\chi_{n}^{2}$. It has been found that $\lambda$ does not get very large, especially in the vicinity of the minimum.

The properties of the Taylor series correction $\delta_{\mathrm{t}}$ in equation (11) make it scaled invariant under linear transformation whereas the properties of the gradient methods are not. Thus an appropriate method for scaling the $\beta$ space leads to the transformation of the matrices $B$ and $E$ in step 4. Then the new step size $\delta$ is calculated using the equations in step 6. Marquardt (1963) points out that this choice of scaling also improves the numerical aspects of the computing procedure.

\begin{abstract}
Résumé
Estimation de paramètres géométriques pour la tomographie à faisceau en éventail.

La qualité de l'image tomographique dépend de la détermination précise des paramètres géométriques qui référencent le système de détection par rapport au système de coordonnées en imagerie transaxiale. En géométrie parallèle, la projection du centre de rotation sur le plan de détection image définit complètement la géométrie tomographique. En complément de la projection du centre de rotation sur le détecteur, la géométrie de faisceau en éventail nécessite deux autres paramètres: la longueur de focalisation et la projection du point de focalisation sur le détecteur. Ces paramètres sont liés au détecteur de façon beucoup plus complexe que ne l'est le centre de rotation projeté nécessaire en géométrie parallèle. Jusqu'ici aucune méthode n'a été développée pour estimer les paramètres géométriques d'un système de détection à faisceau en éventail. Les auteurs présentent une méthode d'estimation de ces paramètres à partir des centroides des projections mesurées d'un point source en utilisant l'algorithme d'estimation non linéaire dû à Marquardt. La technique est appliquée aux données de la tomographie d'émission par photon unique (SPECT) obtenues avec une gamma caméra tournante équipée d'un collimateur à faisceau en éventail. Les paramètres peuvent être déterminés très rapidement dans un environment clinique. Les images reconstruites ne montrent pas d'artéfacts ou de pertes de résolution caractèristiques de paramètres géométriques déterminés de façon inexacte.
\end{abstract}

\title{
Zusammenfassung
}

Bestimmung der geometrischen Parameter bei Fächerstrahlgeometrie.

Die Qualität tomographischer Bilder hängt ab von der genauen Bestimmbarkeit der geometrischen Parameter, die das Detektorsystem in Bezug setzen zu dem transaxialen abbildenden Koordinatensystem. Bei paralleler Geometrie sind die Geometrieparameter vollständig bestimmt durch die Projektion des Rotationsmittelpunktes auf die Detektorebene. Zusätzlich zu dieser Projektion sind bei Fächerstrahlgeometrie zwei weitere Parameter erforderlich, die Fokuslänge und die Projektion des Fokuspunktes auf die Detektorebene. Die Beziehung dieser Parameter zu dem physikalischen Detektor ist komplizierter als die des projizierten Rotationsmittelpunktes bei paralleler Geometrie. Bisher wurde noch kein Verfahren zur Bestimmung der geometrischen Parameter eines Fächerstrahl-Detektor-Systems entwickelt. Deshalb wird hier eine Methode vorgestellt zur Bestimmung dieser Parameter aus den Mittelpunkten gemessener Projektionen einer Punktquelle mit Hilfe der nicht-linearen Schätzalgorithmen von Marquardt. Das Verfahren wurde angewendet bei Einzel-Photon-Emissionscomputertomographie(SPECT)-Daten von einer rotierenden Gammakamera unter Verwendung eines Fächerstrahlkollimators. Die Parameter können vor Ort in der Klinik sehr schnell bestimmt werden. Die entsprechend rekonstruierten Bilder zeigen keine Bildartefakte oder Auflösungsverschlechterung, wie dies bei weniger genau bestimmten Geometrieparametern der Fall ist. 


\section{References}

Deutsch R 1965 Estimation Theory (Englewood Clifts, NJ: Prentice-Hall)

Dreike P and Boyd D P 1976 Comput. Graphics Image Process. 5 459-69

Gullberg G T 1979 Comput. Graphics Image Process. $1030-47$

Gullberg G T, Crawford C R and Tsui B M W 1986 IEEE Trans. Med. Imag. MI-5 23-9

Herman G T and Naparstek A 1977 SIAM J. Appl. Math. 3 511-33

Horn B K P 1979 Proc. IEEE 67 1616-23

Jaszczak R J, Chang L-T and Murphy P H 1979 IEEE Trans. Nucl. Sci. NS-26 610-8

Jaszczak R J, Coleman R E and Whitehead F R 1981 IEEE Trans. Nucl. Sci. NS-28 69-80

Jaszczak R J, Floyd C E Jr, Manglos S H, Greer K L and Coleman R E 1986 Med. Phys. 13 484-9

Keyes J W, Rogers W L, Clinthorne N H et al 1982 Radioaktive isotope in Klinik und Forschung 15 Band ed R Hofer and H Bergmann (Vienna: Egermann) pp 529-37

Kijewski M and Judy P 1983 Med. Phys. 10 169-75

Lim C B, Chang L-T and Jaszczak R J 1980 IEEE Trans. Nucl. Sci. NS-27 559-68

Marquardt D W 1963 SIAM 11 431-41

Shepp L A, Hilal S K and Schultz R A 1979 Comput. Graphics Image Process. 10 246-55

Todd-Pokropek A E 1982 Radioaktive isotope in Klinik und Forschung 15 Band ed R Hofer and H Bergmann (Vienna: Egermann) pp 539-48

Tsui B M W, Gullberg G T, Edgerton E R, Gilland D R, Perry J R and McCartney W H 1986 J. Nucl. Med. 27 810-9

Weinstein F S 1980 J. Opt. Soc. Am. 70 931-5 